\title{
Brillouin Gain Spectrum Characterization in Single-Mode Optical Fibers
}

\author{
Marc Niklès, Luc Thévenaz, and Philippe A. Robert
}

\begin{abstract}
A novel method for Brillouin gain spectrum measurements in optical fibers is presented. It is based on the pump and probe technique with the specificity to use a single laser source together with an external modulator to generate the interacting lightwaves. The high accuracy and inherent stability of the technique makes it suitable for calibration and reference measurements. Different fibers with different refractive index profiles have been tested and characterized. The problem of the evolution of the polarization of the interacting waves is addressed in the article and a polarization insensitive determination of the actual Brillouin gain coefficient is made possible through two successive measurements with different polarizations. The effects of strain and temperature on the Brillouin gain spectrum are also fully characterized.
\end{abstract}

Index Terms-Brillouin scattering, optical fiber measurements, optical fiber scattering, strain measurements, temperature measurements.

\section{INTRODUCTION}

$\mathbf{T}$ HE spectacular success of fiber optics communication originates from the development of low-loss single-mode optical fibers together with high power light sources. Since the breakthrough of silica optical fibers, there has been a continuing interest in the development of long-distance optical communication systems. In order to fully take advantage of the available bandwidth of the optical fibers, several channels can be multiplexed on the same fiber, by using narrow linewidth lasers. Furthermore, the power of optical transmitters are continuously increased to extend the repeater spacing. In that scheme, it has rapidly been observed that the nonlinearities of the silica medium would place an ultimate limit in the capabilities of the optical fibers [1]. Among the nonlinear effects, stimulated Brillouin scattering (SBS) introduces a strong additional loss as soon as the launched power reaches a definite level. For a 10-km fiber optics link, the SBS threshold is in the order of $10 \mathrm{~mW}$ for a $\mathrm{CW}$ laser light, this threshold being even lower for smaller core fibers such as dispersion shifted fibers. However this figure would be different when the light is modulated at frequencies higher than the Brillouin gain spectrum bandwidth. There have been a few attempts to reduce the importance of SBS, by either increasing the signal spectral width by phase modulating the signal, or by introducing nonuniformities by stressing the optical fiber [2].

Manuscript received October 3, 1996; revised July 14, 1997. This work was supported by the Swiss Telecom PTT, PSEL funding and Cossonay Cortaillod Cables.

The authors are with the Laboratory of Metrology, Swiss Federal Institute of Technology (EPFL), Lausanne 1015 Switzerland.

Publisher Item Identifier S 0733-8724(97)07560-9.
Beside setting a limitation for fiber optics communication, SBS has been advantageously utilized in the past few years for different purposes: a) optical fiber characterization [3], [4], b) optical amplification [5], c) distributed attenuation measurements along an optical link [6], d) distributed tensile strain and temperature measurements [7], [8].

The SBS amplifier makes use of the $40 \mathrm{MHz}$ bandwidth of the Brillouin gain to cancel the fiber propagation loss for the carrier in coherent optical transmission and for channel selection in FDM systems. Brillouin spectroscopy brings an elegant and nondestructive tool for fiber characterization. A precise measurement of the Brillouin gain spectrum (BGS) allows an identification of the core content and its density and a determination of geometrical parameter such as the fiber diameter. SBS also provides a way to obtain stronger optical powers than the traditional optical time domain reflectometer (OTDR). Finally the dependence of the Brillouin frequency shift on environmental quantities makes SBS very attractive for sensing applications, such as monitoring strains in installed fiber cables and distributed temperature measurements. For all these applications the Brillouin gain spectrum (BGS) contains important information, such as the Brillouin frequency shift $\nu_{B}$, the Brillouin spontaneous linewidth $\Delta \nu_{B}$, and the linear gain coefficient $g_{0}$, that all depend on environmental quantities. The BGS parameters also provide key information for the determination of the SBS threshold in actual fiber links.

The first observation of the Brillouin lines in bulk silica was made as early as 1950 [9]. More recently, measurements of the BGS were performed on optical glasses by using a single mode Argon laser [10]. These experiments provide a set of information on the spontaneous BGS width and on the associated damping process of sound waves. To date different experimental setups have been proposed to measure the BGS in optical fibers. In 1979 Rowell et al. reported the measurement of the spontaneous BGS in short pieces of single mode fiber at a wavelength of $514.5 \mathrm{~nm}$, using a Fabry-Perot spectrum analyzer [11]. More recent measurements performed with an heterodyne-detection technique by mixing the spontaneously scattered light with the laser light indicates that the Brillouin frequency shift depends on the core doping concentration [12]. The most widely used technique to date is the so-called pump and probe technique [13]. It is based on two separate light sources: the first is used to pump the medium whereas the second generates a frequency tunable probe signal. The BGS is determined by measuring the amplification of the probe when the frequency difference between the pump and the probe corresponds to the Brillouin frequency shift. The 
important drawbacks are the need of two distinct sources to generate the pump and probe signals and the microwave signal processing to determine their frequency difference. This results in several experimental constraints that are difficult to achieve, such as simultaneous power and frequency stability of the two lasers together with low drift, narrow linewidth, and accurate tunability within the Brillouin gain bandwidth.

The purpose of this paper is twofold. On one hand, it presents a highly accurate technique for BGS measurements in optical fibers, and on the other hand, it investigates the characteristics of SBS in optical fibers. After a short general presentation of SBS in Section II, the procedure of the measuring technique is described in Section III. The originality of the method relies on the use of only one single frequency laser and of an external guided-wave modulator. Then the problem of the relative polarization of the pump and probe waves is addressed in Section IV and an elegant solution to the problem is presented. Results of the measurements performed on different optical fibers are presented in Section V. Finally the BGS parameters dependence on temperature and strain has also been investigated and is reported in Sections VI and VII.

\section{BRILLOUIN FREQUENCY SHIFT AND BRILlOUIN GAIN}

Brillouin scattering results from the scattering of light by sound waves. Thermally excited acoustic waves (acoustic phonons) produce a periodic modulation of the refractive index. Brillouin scattering occurs when light is diffracted backward on this moving grating, giving rise to frequency shifted Stokes and anti-Stokes components. This process can be stimulated when the interferences of the laser light and the Stokes wave reinforce the acoustic wave through electrostriction. Since the scattered light undergoes a Doppler frequency shift, the Brillouin shift $\nu_{B}$ depends on the acoustic velocity and is given by

$$
\nu_{B}=\frac{2 n V_{a}}{\lambda}
$$

where $V_{a}$ is the acoustic velocity within the fiber, $n$ is the refractive index and $\lambda$ the vacuum wavelength of the incident lightwave. The strong attenuation of sound waves in silica determines the shape of the BGS. Actually, the exponential decay of the acoustic waves results in a gain $g_{B}(\nu)$ presenting a Lorenzian spectral profile [10].

$$
g_{B}(\nu)=g_{0} \frac{\left(\Delta \nu_{B / 2}\right)^{2}}{\left(\nu-\nu_{B}\right)^{2}+\left(\Delta \nu_{B / 2}\right)^{2}}
$$

where $\Delta \nu_{B}$ is the full-width at half maximum (FWHM). The BGS peaks at the Brillouin frequency shift $\nu_{B}$, and the peak value is given by the Brillouin gain coefficient $g_{0}$

$$
g_{B}\left(\nu_{B}\right)=g_{0}=\frac{2 \pi n^{7} p_{12}^{2}}{c \lambda_{p}^{2} \rho_{0} V_{a} \Delta \nu_{B}}
$$

where $p_{12}$ is the longitudinal elasto-optic coefficient, $\rho_{0}$ is the density, $\lambda_{p}$ is the pump wavelength and $c$ is the vacuum velocity of light [14].

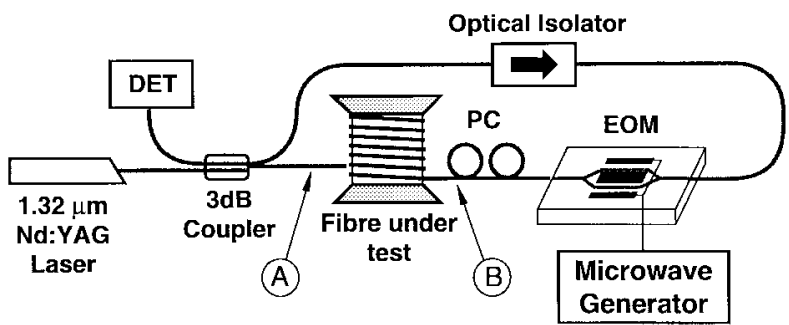

Fig. 1. Experimental setup for Brillouin gain spectrum measurements using an electrooptic modulator (EOM) to generate the interaction waves. ( $\mathrm{PC}=$ polarization controller.)

\section{EXPERIMENTAL SETUP}

The key element of the setup is a $\mathrm{LiNbO}_{3}$ guided-wave intensity modulator. When a single frequency lightwave is modulated at a fixed frequency $f_{m}$ using an external modulator, the modulation gives rise to new frequency lines in the optical spectrum, the so-called modulation sidebands. For pure intensity modulation, the obtained spectrum is symmetrical around the incident lightwave frequency $\nu_{0}$ and the new lines are equally spaced in frequency by $f_{m}$. When the modulation frequency $f_{m}$ is equal to the Brillouin frequency shift $\nu_{B}$, the first-order sidebands can interact with the incident lightwave through SBS process, provided that they propagate in opposite directions. Thus three waves are involved in the interaction, i.e., the incident lightwave at $\nu_{0}$ and the first sidebands at $\nu_{0} \pm f_{m}$. This technique offers determining advantages such as no dependence on the laser frequency drift and no need of a tunable laser source. The BGS can be scanned with ideal accuracy and stability by sweeping the frequency of a microwave generator.

The proper experimental conditions can be achieved using the experimental setup shown in Fig. 1. The optical loop configuration is chosen to make possible opposite directions for the propagation of the laser lightwave at $\nu_{0}$ and of the lines at $\nu_{0} \pm f_{m}$. The light of a $1.32-\mu \mathrm{m}$ diode-pumped single frequency Nd:YAG laser is first split by a coupler. One output of the coupler is directed into the fiber under test while light from the other output is launched into an $\mathrm{LiNbO}_{3}$ intensity electrooptic modulator (EOM). An in-line isolator is inserted inside the loop to avoid interferences and a polarization controller is used to control the polarization states of the interacting waves.

In normal operating conditions, the intensity spectrum at the modulator output is similar to that shown in Fig. 2(a), obtained using a Fabry-Perot analyzer. Better experimental conditions can be achieved when the modulator dc bias voltage is set, so that no light is transmitted in absence of a modulation signal (blocking state). In this case it turns out that in the small modulation regime only the odd-order sidebands are present in the intensity spectrum, as shown in Fig. 2(b). This operation mode has the advantage that only the line at $\nu_{0}$ propagates from $A$ to $B$ through the fiber, while only the sidebands of the modulated signal propagate from $B$ to $A$ and can be detected. The odd higher-order sidebands are also transmitted, but they are separated by a frequency $2 f_{m}$ from the closest lines, so that only the first-order sidebands at $\nu_{0} \pm f_{m}$ can experience 


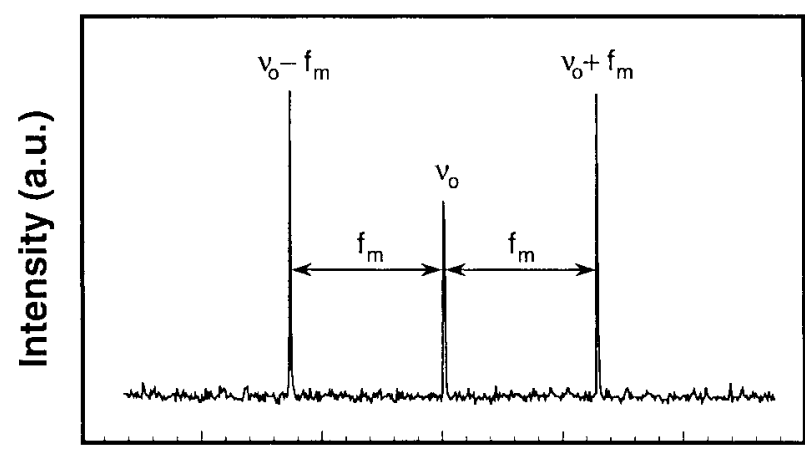

(a)

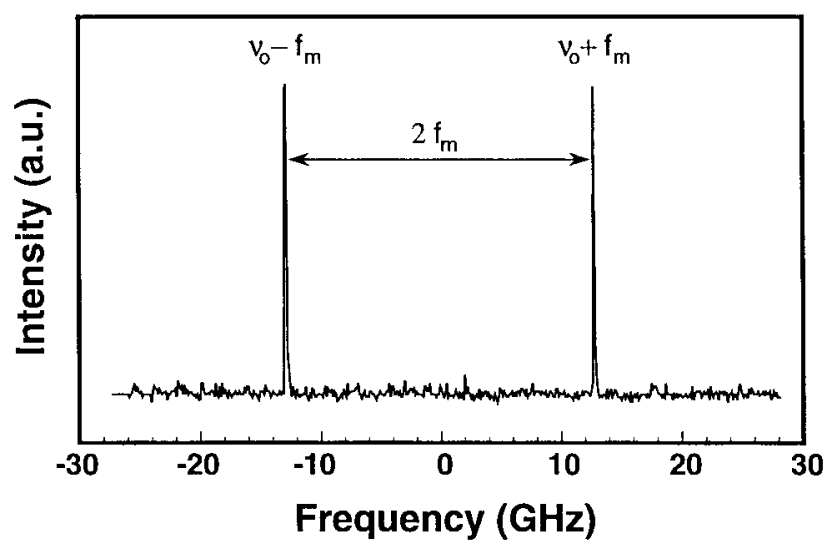

(b)

Fig. 2. Optical intensity spectra scanned with a Fabry-Perot analyzer of a single frequency laser modulated by an electrooptic modulator. (a) Carrier and first order sidebands and (b) suppression of the carrier by properly setting the dc bias on the modulator electrodes.

a stimulated Brillouin scattering through interaction with the counterpropagating laser lightwave at $\nu_{0}$.

The BGS is measured by sweeping the modulation frequency $f_{m}$ in the vicinity of the Brillouin frequency shift $\nu_{B}$ and by detecting the total intensity using a low-frequency detection scheme. When the modulation frequency falls within the BGS, an interaction takes place between waves propagating in opposite directions and the evolution of their intensity is governed by the standard intensity rate equations [14]. The lightwave at frequency $\nu_{0}-f_{m}$, the first lower sideband, is amplified by the line at $\nu_{0}$ and is found to grow exponentially, provided that the intensity at $\nu_{0}$ is much larger and pump depletion can thus be neglected. On the contrary, the lightwave at frequency $\nu_{0}+f_{m}$, the first upper sideband, amplifies the line at $\nu_{0}$. The sideband intensity being very low, the actual gain is very small and the intensity increase at $\nu_{0}$ can be fully neglected. But the large intensity at $\nu_{0}$ makes even the smallest gain to significantly deplete the low intensity sideband at $\nu_{0}+f_{m}$. This process is schematically represented in Fig. 3. Solving the rate equation for this situation is also easy and the intensity at $\nu_{0}+f_{m}$ turns out to decay exponentially. Consequently the two sidebands intensities at points $A$ and $B$ along the fiber are given by

$$
\begin{aligned}
& I_{-1}^{A}=I_{-1}^{B} \exp \left\{g_{B}(\nu) I_{C}^{A} L_{\mathrm{eff}}-\alpha L\right\} \\
& I_{+1}^{A}=I_{+1}^{B} \exp \left\{-g_{B}(\nu) I_{C}^{A} L_{\mathrm{eff}}-\alpha L\right\}
\end{aligned}
$$

where $I_{C}^{A}$ is the intensity at frequency $\nu_{0}, g_{B}(\nu)$ the frequencydependent Brillouin gain, $L_{\text {eff }}=[1-\exp (-\alpha L)] / \alpha$ is the usual effective interaction length for nonlinear effects, $\alpha$ is the fiber loss coefficient and $L$ the total fiber length. Assuming that the two sidebands initially have the same magnitude, $I_{+1}^{B}=I_{-1}^{B}=I_{S}^{B}$, the total detected intensity turns out to be proportional to

$$
I_{\text {out }}=I_{-1}^{A}+I_{+1}^{A}=2 \exp \{-\alpha L\} I_{S}^{B} \cosh \left\{g_{B}(\nu) I_{C}^{A} L_{\text {eff }}\right\} .
$$

The condition of a large intensity at $\nu_{0}$ with respect to the sideband intensities makes possible the decorrelation of the SBS processes experienced by the two sidebands and eventually yields this simple hyperbolic cosine relation.

The BGS has a Lorenzian spectral profile, as mentioned in Section II. Therefore, the dependence of the measured output intensity on the modulation frequency must fit a hyperbolic cosine of a Lorenzian curve. Since the linewidth of all the lines is very narrow $(<500 \mathrm{kHz})$, the resolution of the measurement is excellent. Moreover, the use of only one laser cancels the effects of any frequency drift and consequently improves the resolution on the BGS width determination. Fig. 4 shows the measured BGS of a 700-m standard telecommunication fiber (matched cladding, 3 wt.\% $\mathrm{GeO}_{2}$-core-doped concentration, core diameter $9 \mu \mathrm{m})$. Measurements were performed at room temperature $\left(25^{\circ} \mathrm{C}\right)$ and the optical fiber was wound with no tension to avoid any strain. The agreement between experimental points and the numerical fit of the function given in (6) for a Lorenzian BGS is excellent, as can be seen in Fig. 4. The measured $\Delta \nu_{B}$ is $35.8 \mathrm{MHz}$ and the Brillouin frequency shift $\nu_{B}$ is $12.8138 \mathrm{GHz}$ at a wavelength of $1.32 \mu \mathrm{m}$.

The intensity of the sidebands can be easily controlled by varying the modulation amplitude and is of prime importance in the precise determination of the BGS. Actually, if the sidebands intensity is too large, the effect on the laser lightwave at $\nu_{0}$ can no longer be neglected and the measured BGS is distorted. The depletion of the pump is larger around the maximum of the BGS, decreasing the gain for these frequencies. The shape of the BGS is flattened and this results in an overestimated BGS width. For instance, for a 700-m fiber and a pump power $I_{C}^{A}$ of $20 \mathrm{~mW}$, the sidebands power should not exceed 10 microwatt, as can be seen in Fig. 5.

\section{POLARIZATION ISSUES}

Since SBS originates from the coherent mixing of pump and probe waves, the efficiency of the effect is polarization dependent. This particular aspect of SBS in fibers has been observed by several authors and a polarization averaging technique has been proposed in order to reduce contrast fluctuations due to polarization change in distributed Brillouin gain based sensors [6]. Here we demonstrate that polarization effects in Brillouin gain determination can be exactly canceled out in any situation by performing two successive measurements.

For polarization maintaining fibers the Brillouin gain is maximum for parallel linear polarization aligned to one of the birefringence axis of the fiber, zero for orthogonal linear polarization aligned to the birefringence axis, and $1 / 2$ for linear polarization launched at $45^{\circ}$ of the birefringence axis. 


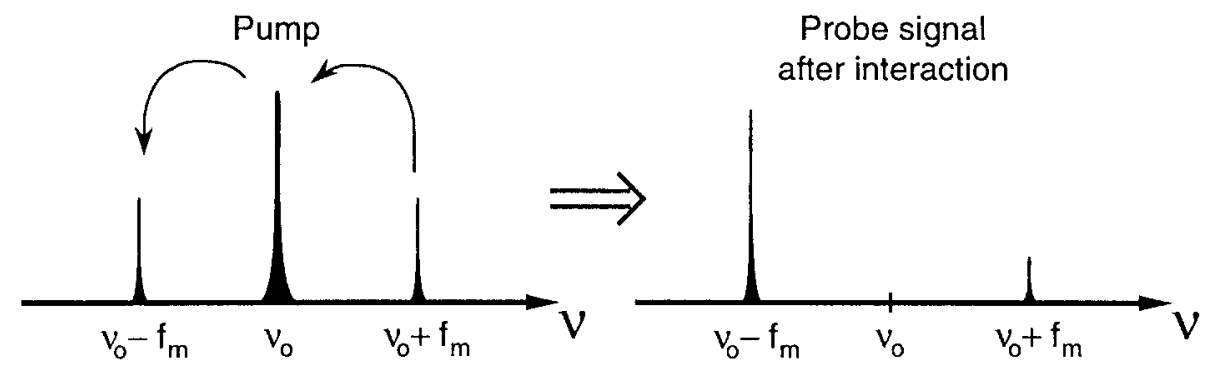

Fig. 3. Schematic diagram of the energy transfer between the pump wave and the two modulation sidebands.

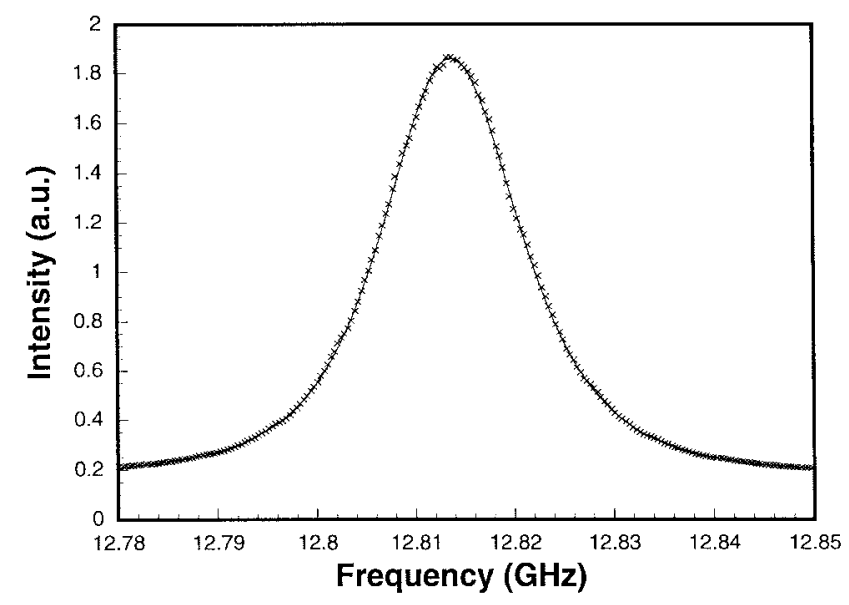

Fig. 4. Brillouin gain spectrum of a 700-m single mode fiber. The experimental data (crosses) perfectly fit the hyperbolic cosine of a Lorenzian function. $\Delta \nu_{B}=35.8 \mathrm{MHz}, \nu_{B}=12.8138 \mathrm{GHz}$.

Deventer et al. showed that the situation is entirely different for low-birefringence fibers [15]. The interaction between pump and probe is maximum when the orientation, the ellipticity and sense of rotation are the same when seen from the same direction. However for counterpropagating waves, if the sense of rotation is the same the handedness of the polarization is the inverse. Therefore the Brillouin gain cannot be zero even if the polarization of the launched probe is orthogonal to the pump in B (Fig. 1), provided that the fiber length exceed a few birefringence beat length and when the polarization is not preserved. Deventer et al. demonstrated that the overall gain in low-birefringence fibers ranges from $1 / 3$ to $2 / 3$ of the Brillouin gain coefficient for a complete polarization scrambling and is not equal to $1 / 2$ as often mentioned in the literature. Starting from this statement we propose a method to measure in any situation the actual Brillouin gain coefficient of a piece of fiber even when the polarization is neither preserved nor completely scrambled. It is based on two successive measurements of the Brillouin gain with two different particular states of polarization. The polarization controller is adjusted so that the contrast is maximum for the first measurement and minimum for the second. The local gain variations due to polarization mismatch between pump and probe can be described by a function of the position $z, \gamma(z)$. Actually $\gamma(z)$ corresponds to the square of the scalar product between the pump and probe polarization vectors and $0<\gamma(z)<1$. If $\gamma(z)$ represents the local polarization mismatch when the gain contrast is maximum, then the local polarization mismatch when the

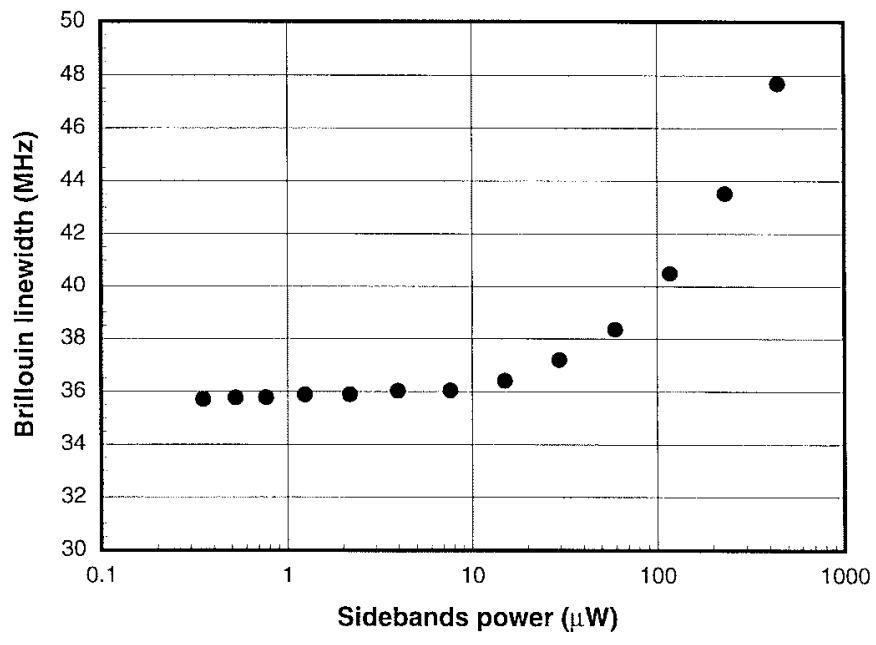

Fig. 5. Measured Brillouin linewidth $\Delta \nu_{B}$ of $700 \mathrm{~m}$ single mode fiber as function of the sidebands power for a carrier power of $20 \mathrm{~mW}$, showing the overestimated width due to pump depletion.

contrast is minimum is given by $1-\gamma(z)$. This property results from the orthogonality of the polarization states in the two situations. The intensity rate equations can therefore be solved for these two particular cases after introducing $\gamma(z)$. Under the assumption that the wavelength separation between the two relevant sidebands is very small and the birefringence is small, the sidebands are in the same state of polarization at any location along the fiber. The detected intensities turn out to be after integrating over the entire fiber length

$$
\begin{aligned}
I_{\mathrm{out}}^{\max } & =2 \exp \{\alpha L\} I_{S}^{B} \cosh \left\{\Gamma g_{B}(\nu) I_{C}^{A} L_{\mathrm{eff}}\right\} \\
& =I_{0} \cosh \left\{\Gamma g_{B}(\nu) I_{C}^{A} L_{\mathrm{eff}}\right\} \\
I_{\mathrm{out}}^{\min } & =2 \exp \{\alpha L\} I_{S}^{B} \cosh \left\{(1-\Gamma) g_{B}(\nu) I_{C}^{A} L_{\mathrm{eff}}\right\} \\
& =I_{0} \cosh \left\{(1-\Gamma) g_{B}(\nu) I_{C}^{A} L_{\mathrm{eff}}\right\}
\end{aligned}
$$

where

$$
\Gamma=\int_{0}^{L} \gamma(z) d z
$$

and

$$
I_{0}=2 \exp (\alpha L) I_{S}^{B}
$$

Finally from (7) and (8), the actual Brillouin gain coefficient of the fiber can be determined by summing the Acosh of the BGS measured in the conditions of maximum and minimum 


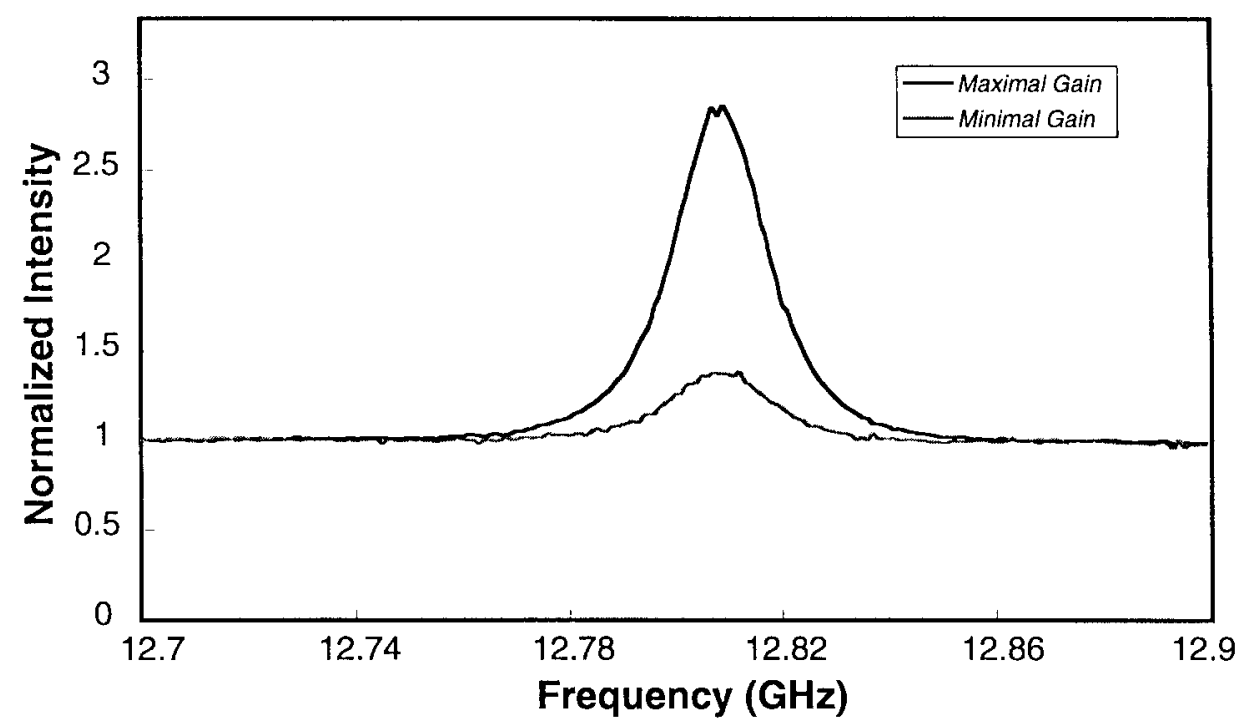

(a)

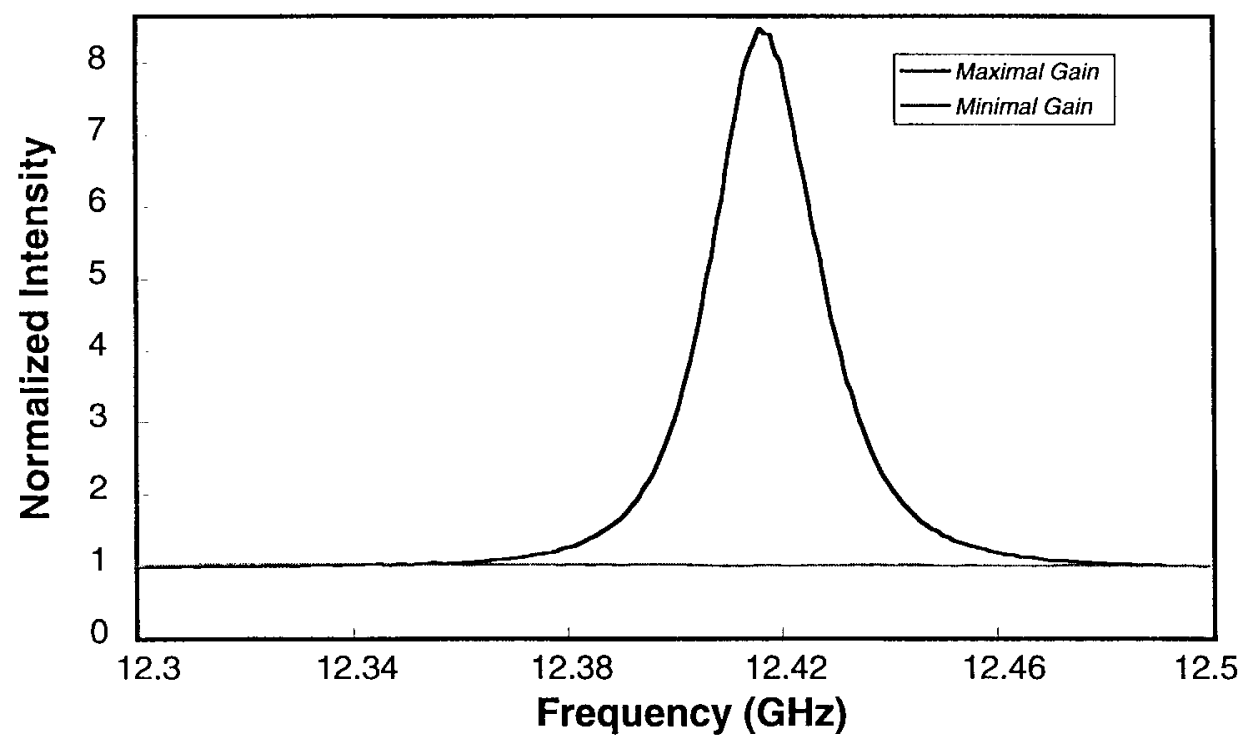

(b)

Fig. 6. Maximum and minimum gain achievable in the measurement of the BGS of (a) a standard telecommunication fiber and (b) a highly birefringent dispersion shifted fiber.

contrast, respectively

$$
A \cosh \left(\frac{I_{\mathrm{out}}^{\min }}{I_{0}}\right)+A \cosh \left(\frac{I_{\mathrm{out}}^{\max }}{I_{0}}\right)=g_{B}(\nu) I_{C}^{A} L_{\mathrm{eff}}
$$

Equation (11) leaves a polarization independent expression for the Brillouin gain coefficient $g_{0}$. Fig. 6(a) shows the measurements of the BGS of a $140 \mathrm{~m}$ standard telecommunication fiber with minimum and maximum achievable contrast. The measured maximum and minimum value of the gain are respectively $67 \%$ and $33 \%$ of the Brillouin gain coefficient $g_{0}=2.6 \times 10^{-12} \mathrm{~m} / \mathrm{W}$, in full agreement with the measured value in polarization maintaining fibers. Fig. 6(b) shows the same measurements performed on $140 \mathrm{~m}$ of highly birefringent dispersion shifted fiber. If the polarizations of the launched waves are linear and parallel to one of the birefringence axis of the fiber, they remain unchanged. Therefore the contrast is zero if the polarizations are orthogonal and maximum if the polarizations are parallel.

\section{EXPERIMENTAL RESULTS}

First a set of different single-mode optical fibers with $\mathrm{GeO}_{2}$-core concentration ranging from 0 to 17.65 wt. $\%$ was measured. The length of the different fibers was in the 100 to $200 \mathrm{~m}$ range and a special care was taken in the conditioning of the different fiber samples in order to insure a perfect uniformity in the scattering characteristics. As a matter of fact, if the parameters determining the BGS can be assumed to be constant over the whole fiber length, the intrinsic BGS of the fiber can be determined. Furthermore the samples were placed in a temperature controlled environment and were wound by hand to avoid any strain. Regular mechanical winding 
TABLE I

Summary of the Measurements Performed on Different Single Mode Optical Fibers, Together with Some Fibers Characteristics and Calculated Gain. Values for Bulk Silica Are Extrapolated From Reported Measurements

\begin{tabular}{|c|c|c|c|c|c|c|c|}
\hline $\begin{array}{c}\mathrm{GeO}_{2} \text {-core } \\
\text { content (wt.\%) }\end{array}$ & $\begin{array}{l}\text { Brillouin shift } \\
\text { (GHz) }\end{array}$ & $\begin{array}{c}\text { Linewidth } \\
(\mathrm{MHz})\end{array}$ & $\begin{array}{c}\text { Spot size } \\
(\mu \mathrm{m})\end{array}$ & $\begin{array}{l}\text { Max. gain } \\
\text { (\% Total gain) }\end{array}$ & $\begin{array}{c}\text { Min. gain } \\
\text { (\% Total gain) }\end{array}$ & $\begin{array}{c}\text { go/Aeff } \\
\left(1 /\left(m^{*} W\right)\right)\end{array}$ & $\begin{array}{c}\text { Peak gain go } \\
(\mathrm{m} / \mathrm{w})\end{array}$ \\
\hline Bulk Silica & 13.1 & 23 & & & & & $5.00 \mathrm{E}-11$ \\
\hline 0 (F-doped cladding) & 12.9896 & 50.74 & 4.4 & 65 & 35 & 0.28 & $1.73 \mathrm{E}-11$ \\
\hline 2.65 & 12.8527 & 35.5 & 5.04 & 67 & 33 & 0.33 & $2.62 E-11$ \\
\hline 2.94 & 12.7974 & 37.5 & 4.64 & 66 & 34 & 0.34 & $2.27 \mathrm{E}-11$ \\
\hline 2.94 & 12.8434 & 31.29 & 4.64 & 72 & 28 & 0.45 & $3.04 \mathrm{E}-11$ \\
\hline 2.94 & 12.8082 & 35.5 & 4.82 & 67 & 33 & 0.36 & 2.60E-11 \\
\hline 4.71 & 12.7191 & 34.5 & 4.16 & 76 & 24 & 0.48 & $2.62 \mathrm{E}-11$ \\
\hline 6.18 & 12.3882 & 40.4 & 3.44 & 77 & 23 & 0.60 & $2.24 \mathrm{E}-11$ \\
\hline 6.50 & 12.4178 & 45.94 & 3.2 & 100 & 0 & 0.67 & 2.15E-11 \\
\hline 8.24 & 12.3343 & 42.1 & 2.96 & 87 & 13 & 0.59 & $1.63 \mathrm{E}-11$ \\
\hline 9.41 & 12.134 & 44.2 & 3 & 67 & 33 & 1.08 & $3.05 E-11$ \\
\hline 10.00 & 12.054 & 45 & 3.1 & 100 & 0 & 0.83 & $2.51 \mathrm{E}-11$ \\
\hline 17.65 & 11.504 & 55 & 1.84 & 94 & 6 & 1.82 & $1.93 \mathrm{E}-11$ \\
\hline
\end{tabular}

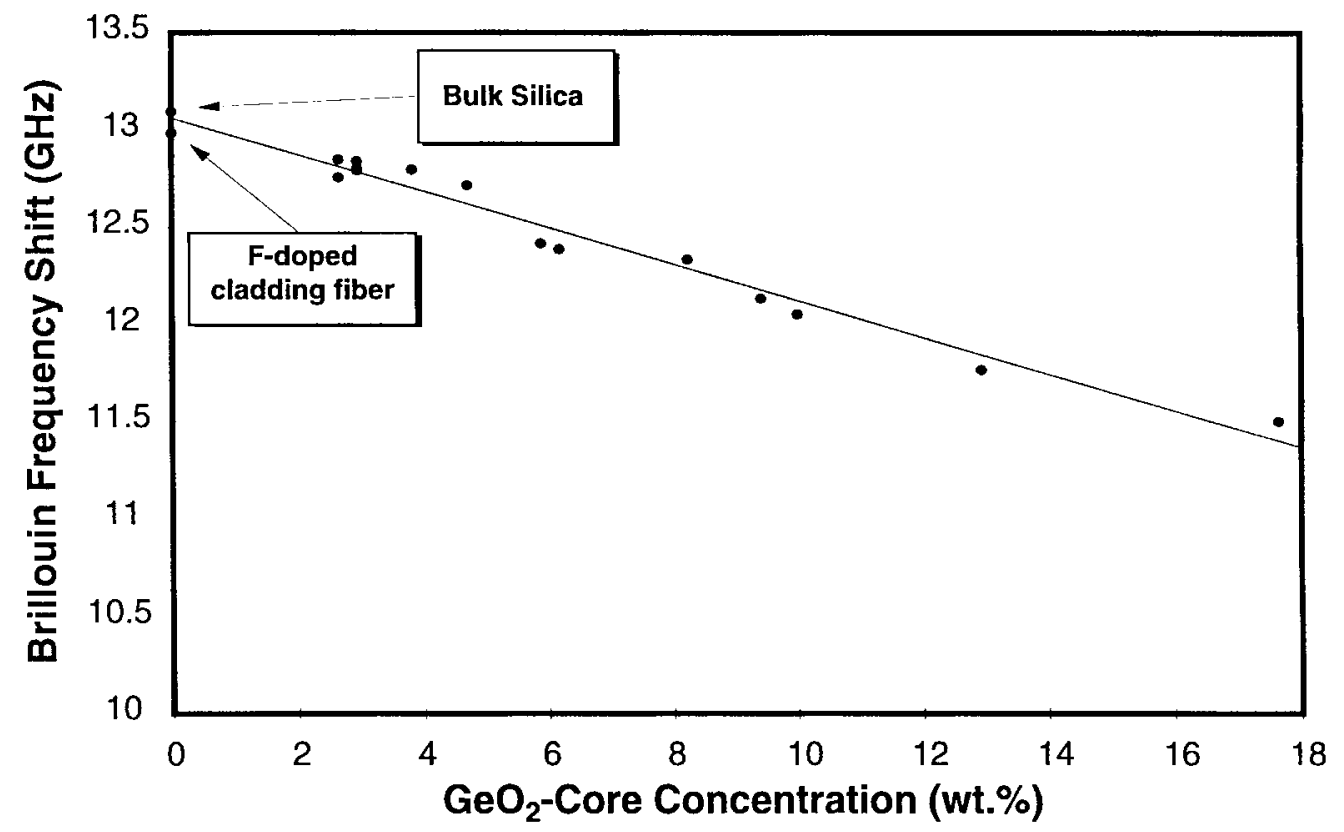

Fig. 7. Measured dependence of the Stokes frequency shift $\nu_{B}$ on the $\mathrm{GeO}_{2}$-core concentration.

induces stresses that were observed to bias the measurements by causing an nonhomogeneous broadening of the gain curve.

Table I summarizes the measurements performed on theses fibers. The Brillouin frequency shifts range from $11.5 \mathrm{GHz}$ to $13 \mathrm{GHz}$ at $1.32 \mu \mathrm{m}$ and a change of $-94 \mathrm{MHz} / \mathrm{wt} . \%$ is found between Brillouin shift and $\mathrm{GeO}_{2}$-core concentration, as shown in Fig. 7, confirming and specifying previously reported results [12], [16]. The points on the vertical axis correspond to the reported value for bulk silica and to a pure silica core fiber with an $F$-doped cladding respectively. For both cases the agreement is excellent with the extrapolated value for pure silica.
A change of $1.4 \mathrm{MHz} / \mathrm{wt} . \%$ was found between Brillouin linewidth $\Delta \nu_{B}$ and $\mathrm{GeO}_{2}$-core concentration, as shown in Fig. 8. The bulk silica and $F$-doped values were not taken into account for the determination of this relationship. The extrapolated value for $\Delta n=0$ is close to the bulk silica value, but the agreement is not perfect. This may be due to excess phonon damping occurring in the fiber, though the confidence level of the measurements made in bulk silica is not known. As a matter of fact, the main difference between SBS in optical fibers and in bulk silica deals with the concept of acoustic modes. Rowell et al. were the first to describe guided fiber acoustic modes involved in SBS [11]. A theory for longitudinal 


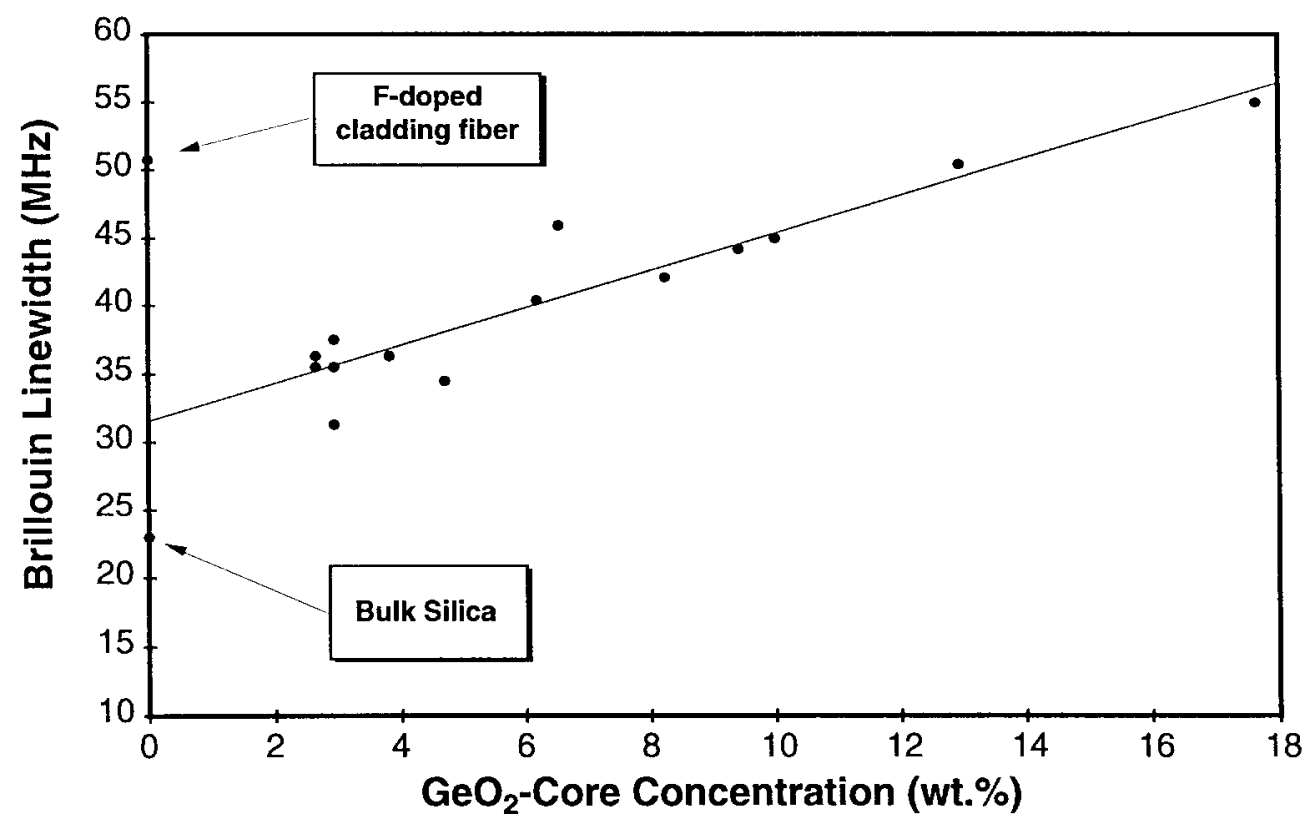

Fig. 8. Measured dependence of the Brillouin linewidth $\Delta \nu_{B}$ on the $\mathrm{GeO}_{2}$-core concentration.

acoustic modes in $\mathrm{GeO}_{2}$ doped fibers was presented by Shibata et al. [16]. It is the nature and concentration of doping in the fiber that determines the guiding properties of the fiber core for acoustic waves. Therefore, while being a single mode optical waveguide, an optical fiber can be a multimode acoustic waveguide, since the acoustic propagation constant is twice larger than the optical propagation constant. Evaluating the acoustic-optical modal overlap factor shows that longitudinal acoustic modes are dominant toward shear acoustic modes and the major gain of SBS comes from the lowest order acoustic mode $\mathrm{LP}_{01}$. In the case of $\mathrm{GeO}_{2}$-core-doped fibers, the addition of germanium in the core decreases both optical and acoustic phase velocities, resulting in a waveguide for both acoustic and optical waves. Out of the 15 tested fibers two dispersion shifted fibers showed a second peak in the BGS lying 600 $\mathrm{MHz}$ apart of the main peak, giving evidence of a higher order guided longitudinal acoustic mode. The magnitude of this second peak was in both cases inferior to one third of the magnitude of the main peak. The pure silica core fiber ( $F$ doped cladding fiber) presents a larger linewidth compared to $\mathrm{GeO}_{2}$-core-doped fibers, as can be seen in Fig. 8. This broadening is attributed to antiguiding properties of the core for acoustic waves, due to the addition of fluoride in the cladding, resulting in an enhanced acoustic loss accordingly.

These measurements provide with a high confidence level a $35 \mathrm{MHz}$ value for the Brillouin FWHM linewidth of standard telecommunication fibers (3 wt. $\% \mathrm{GeO}_{2}$-core-doped) at 1.32 $\mu \mathrm{m}$. Finally the Brillouin gain coefficient $g_{0}$ was calculated from the maximum of the BGS after measurements of pump power and spot size $\omega_{0}$. The effective cross section $A_{\text {eff }}$ was determined using the classical formula $A_{\text {eff }}=\pi \omega_{0}^{2}$. A quite important fluctuation in the gain evaluation $\left(1.4-2 \times 10^{-11}\right.$ $\mathrm{m} / \mathrm{W}$ ) is observed but there is no clear correlation between gain value and core doping concentration. This confirms the assumption that Brillouin interaction predominantly takes

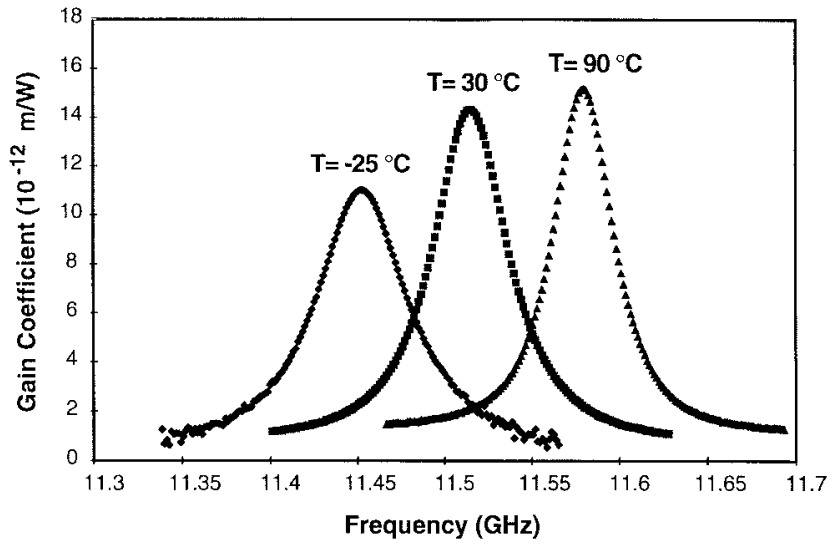

Fig. 9. Brillouin gain spectrum of a single-mode fiber measured at different temperatures showing the effects of temperature on the central frequency and the linewidth and the peak gain.

place within the core, so that the effective cross section $A_{\text {eff }}$ must be weighted by the overlap integral between mode field distribution and core index profile [12]. Actually the highest gain is obtained for a fiber with no dip and a very uniform core index distribution, and lowest gain for a triangular profile.

The difficulty to exactly determine the effective cross section $A_{\text {eff }}$ makes the power gain coefficient $g_{0} / A_{\text {eff }}$, shown in Table I, more suitable for fiber characterization. Overall amplification can be calculated directly from pump power and comparative measurements are possible without further calculations using additional parameters (spot size, index profile).

\section{TEMPERATURE EFFECTS}

The effect of temperature on the BGS can be seen on the measurements shown in Fig. 9. The linearity of the central frequency dependence on temperature claimed by all authors is here confirmed to a high confidence level over a wide temperature range, as shown in Fig. 10(a). The slope coefficient 


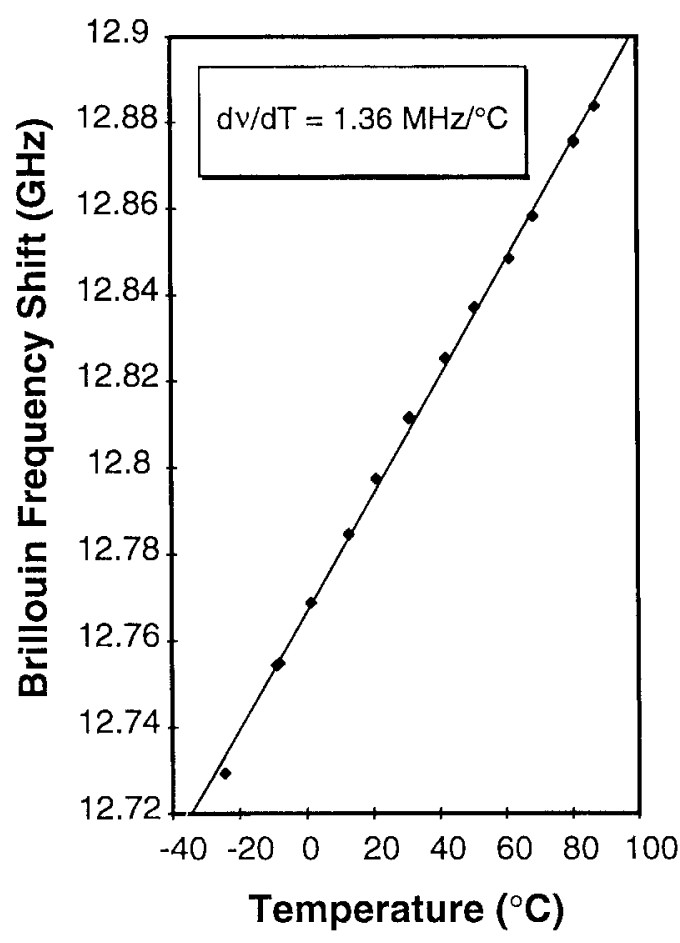

(a)

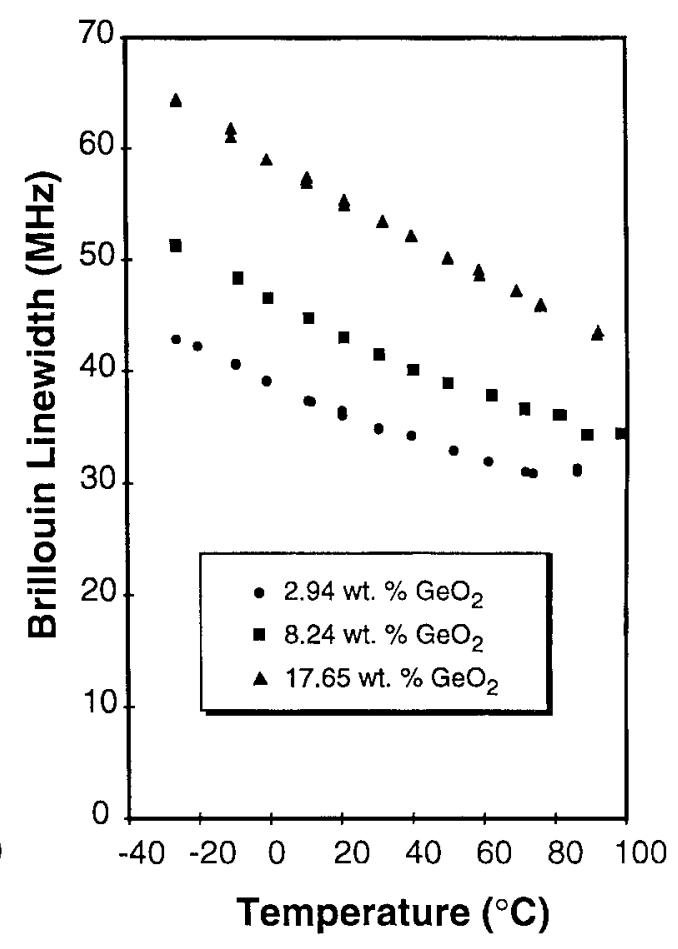

(b)

Fig. 10. Central frequency (a) and linewidth (b) of the Brillouin gain spectrum as a function of temperature. Linewidth measurements are shown for fibers with different $\mathrm{GeO}_{2}$-core content.

of $1.36 \mathrm{MHz} /{ }^{\circ} \mathrm{C}$ at $1.32 \mu \mathrm{m}$ slightly decreases for a higher $\mathrm{GeO}_{2}$ core content. All fiber samples are only coated with a $250-\mu \mathrm{m}$ acrylate microjacket, so that the coating influence is expected to be kept below $0.1 \mathrm{MHz} /{ }^{\circ} \mathrm{C}$ [17].

A new interesting feature is the decrease of the Brillouin linewidth with temperature, as shown in Fig. 10(b). The dependence is not linear and converges to a constant value at higher temperature for all fiber types. According to works achieved using fused quartz [18], the phonon absorption peaks at a temperature close to $100 \mathrm{~K}$ where the Brillouin linewidth must be maximum, accordingly. The observed linewidth decrease with temperature possibly represents the upper tail of the absorption peak. The broader BGS observed at room temperature for fiber with higher $\mathrm{GeO}_{2}$-core content is thus due to either a higher absorption peak or a higher temperature for the maximum absorption. Measurements of Brillouin linewidth in the 100 $\mathrm{K}$ temperature range are necessary to discriminate between these two possibilities.

The maximum gain increases with temperature and should be proportional to $\Delta \nu^{-1}$, so that the Brillouin gain spectrum integrated over all frequencies remains constant. The product $g_{0} \times \Delta \nu$ was checked to be temperature-independent, so that it demonstrates the invariance on temperature of the electrostrictive strength responsible for the stimulation of Brillouin scattering. It turns out that the gain increase with temperature is only due to its spectral narrowing and thus phonon smaller absorption.

\section{STRAIN EFFECTS}

The acoustic velocity $V_{a}$ is strain-dependent in silica fibers [18], so that the BGS central frequency $\nu_{B}$ is expected to vary

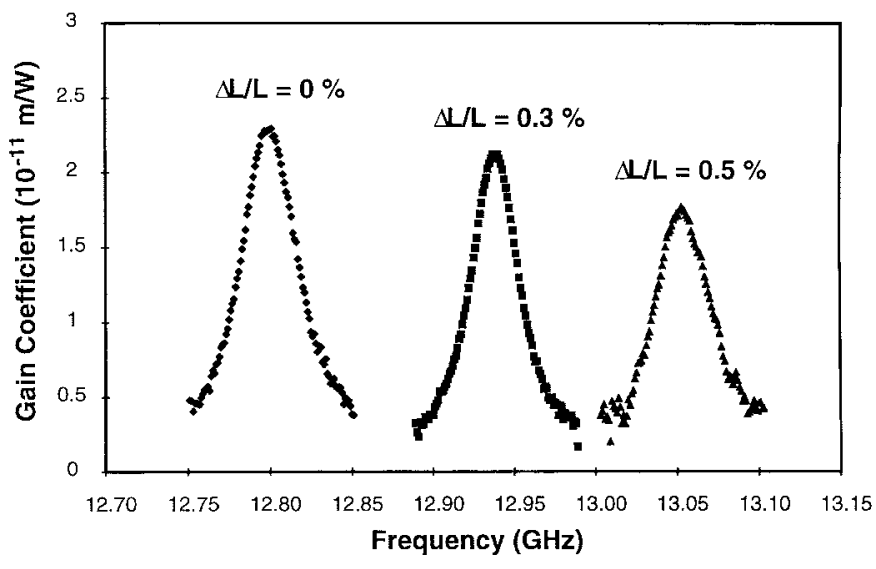

Fig. 11. Brillouin gain spectrum of a single mode fiber measured for different fiber elongation, showing the effects of strain on the central frequency, the linewidth and the peak gain.

when the fiber is under tension. Calibrated elongation was applied to $60 \mathrm{~m}$ fiber samples and the BGS were measured up to the fiber breaking point ( $~ 1 \%$ elongation) using our set-up.

The global effect of strain on BGS is shown in Fig. 11. As previously reported [19], the central frequency $\nu_{B}$ shows a strong dependence on strain of several tens of $\mathrm{MHz}$ for a $1 \%$ elongation. The high accuracy of the experimental set-up clearly demonstrates that the linewidth $\Delta \nu$ remains unchanged with strain, while the gain $g_{0}$ decreases for elongation close to the breaking point. The excellent linearity of the central frequency $\nu_{B}$ on strain and the invariance of the linewidth $\Delta \nu$ is confirmed in the detailed measurement shown in Fig. 12. 


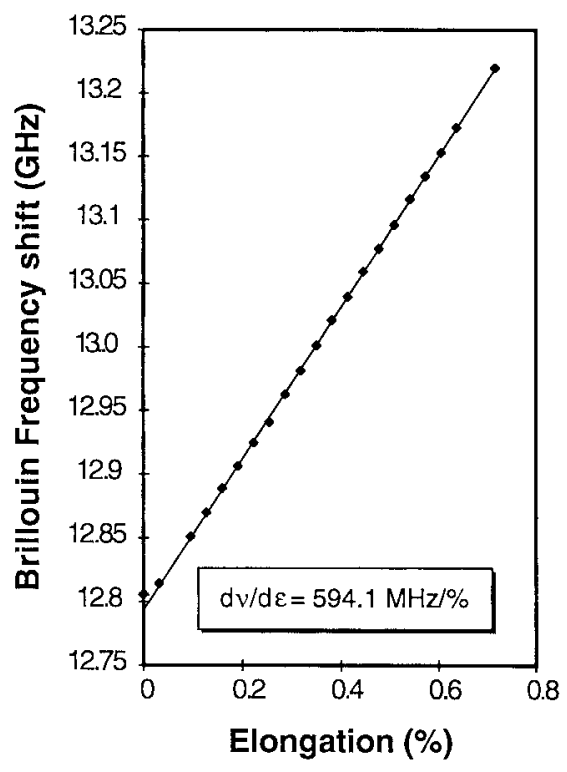

(a)

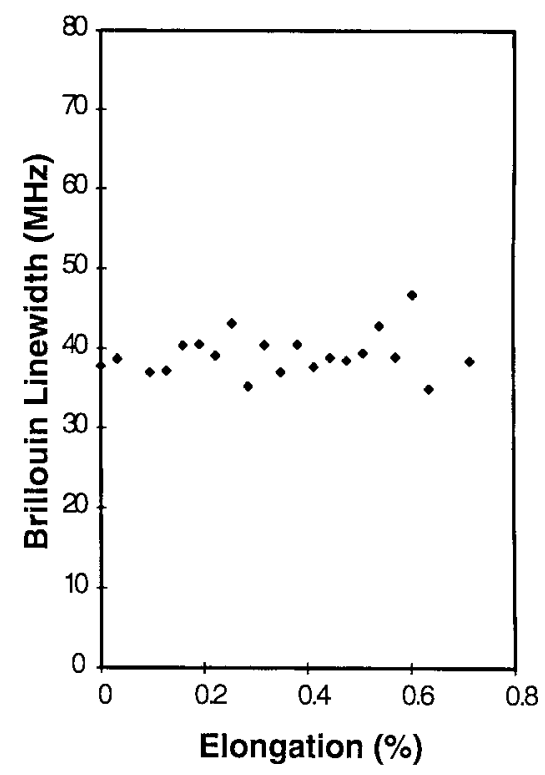

(b)

Fig. 12. Central frequency (a) and linewidth (b) of the Brillouin gain spectrum as a function of elongation.

The measured normalized slope coefficient $\left(1 d \nu_{B}\right) /\left(\nu_{B} d \varepsilon\right)$ ranges from 4.64 to 4.73 for standard fibers at $1320 \mathrm{~nm}$. This value is slightly higher than the previously reported 4.4 coefficient [19], resulting in a central frequency shift very close to $600 \mathrm{MHz}$ per percent elongation at $1.32 \mu \mathrm{m}$. This coefficient was checked to be negligibly dependent on the fiber jacket type, confirming the great potentiality of BGS analysis for monitoring strains actually experienced by fibers.

The gain coefficient $g_{0}$ depends on many structural quantities such as material density $\rho$ and refractive index $n$, that may change in a nonnegligible fraction for high strain. This may explain the gain decrease for strain in the percent range. It is remarkable that the central frequency dependence on strain keeps linear under such extreme conditions.

\section{CONCLUSION}

In conclusion a novel method for BGS measurement of optical fibers has been presented. The use of only one laser source together with an EOM makes possible the generation of an inherent high stability jitters-free probe signal, as well as accurate setting of the relative pump and probe levels. Measurements performed with different sidebands levels clearly shows that a very low pump depletion is necessary for accurate gain linewidth determination.

In addition, repetitive measurements of BGS of the same fiber in controlled environment show a standard deviation of $100 \mathrm{kHz}$ on the Brillouin frequency shift and $200 \mathrm{kHz}$ on the FWHM of the BGS. This represents the most accurate measurement of BGS in optical fibers to our knowledge and this technique is currently used to perform calibration measurement on the dependence of the BGS on environmental quantities. In addition polarization-independent gain measurements have been performed using a new original method. The three parameters fully characterizing the BGS are measured with a high accuracy under different temperature and strain conditions. Thanks to these measurements one can conclude that the Brillouin central frequency $\nu_{B}$ depends very linearly on either temperature or strain over an extended range $\left(-30{ }^{\circ} \mathrm{C}\right.$ $\left.<T<100^{\circ} \mathrm{C}, 0<\varepsilon<1 \%\right)$. The effects of temperature and strain cannot be discriminated by just measuring the central frequency $\nu_{B}$. The BGS linewidth is temperature-dependent owing to the presence of an absorption peak at low temperature $(\sim 100 \mathrm{~K})$, while an applied strain leaves the linewidth unchanged. The linewidth measurement in addition to the central frequency determination could therefore decorrelate temperature from strain effects, provided that no significant change on these quantities occurs within the measurement spatial resolution.

The generation of the probe signal using a modulator may bring many more advantages in addition to those presented here, such as frequency-domain coding for a more sophisticated and sensitive detection and time-domain coding to address distinct positions along the fiber. Distributed Brillouin gain spectrum measurements can then be performed, yielding a very efficient tool for installed fiber optic cables monitoring. These more advanced modulation schemes are currently investigated in our laboratory [20]-[22].

\section{ACKNOWLEDGMENT}

The authors would like to thank F. Cochet from Cabloptic for kindly providing fiber samples and technical data and C. Gabioud for scientific support.

\section{REFERENCES}

[1] A. R. Chraplyvy, "Limitations on lightwave communications imposed by optical-fiber nonlinearities," J. Lightwave Technol., vol. 8, p. 1548, 1990.

[2] N. Yoshizawa, T. Horigushi, and T. Kurashima, "Proposal for stimulated Brillouin scattering suppression by fiber cabling," Electron. Lett., vol. 27, p. 1100, 1991.

[3] T. C. Rich and D. A. Pinnow, "Evaluation of fiber optical waveguides using Brillouin spectroscopy," Appl. Opt., vol. 13, p. 1376, 1974. 
[4] M. Ohashi, N. Shibata, and K. Shiraki, "Fiber diameter estimation based on gided acoustic wave Brillouin scattering," Electron. Lett., vol. 28, p. 900, 1992.

[5] N. A. Olsson and J. P. van der Ziel, "Cancelation of fiber loss by semiconductor laser pumped Brillouin amplification at $1.55 \mu \mathrm{m}$," Appl. Phys. Lett., vol. 48, p. 1329, 1986.

[6] T. Horiguchi and M. Tateda, "Optical-fiber-attenuation investigation using stimulated Brillouin scattering between a pulse and a continous wave," Opt. Lett., vol. 14, p. 408, 1989.

[7] T. Horiguchi, T. Kurashima, and M. Tateda, "A technique to measure distributed strain in optical fibers," IEEE Photon. Technol. Lett., vol. 2, p. 352, 1990

[8] X. Bao, D. J. Webb, and D. A. Jackson, "22-km distributed temperature sensor using Brillouin gain in an optical fiber," Opt. Lett., vol. 18, p. 552, 1993.

[9] R. S. Krishnan, Nature, p. 933, 1950.

[10] D. Heiman, D. S. Hamilton, and R. W. Hellwarth, "Brillouin scattering measurements on optical glasses," Phys. Rev. B, vol. 19, p. 6583, 1979.

[11] N. L. Rowell, P. J. Thomas, H. M. van Driel, and G. I. Stegeman, "Brillouin spectrum of single-mode optical fibers," Appl. Phys. Lett., vol. 34, p. 139, 1979.

[12] R. W. Tkach, A. R. Chraplyvy, and R. M. Derosier, "Spontaneous Brillouin scattering for single-mode fiber characterization," Electron. Lett., vol. 22, p. 1011, 1986.

[13] N. Shibata, R.-G. Waarts, and R. P. Braun, "Brillouin-gain for singlemode fibers having pure-silica $\mathrm{GeO}_{2}$-doped, and $\mathrm{P}_{2} \mathrm{O}_{5}$-doped cores," Opt. Lett., vol. 12, p. 269, 1987.

[14] G. P. Agrawal, Nonlinear Fiber Optics. Boston, MA: Academic, 1989.

[15] M. O. Deventer and A. J. Boot, "Polarization properties of stimulated Brillouin scattering in single-mode fibers," J. Lightwave Technol., vol. 12, p. $585,1994$.

[16] N. Shibata, K. Okamoto, and Y. Azuma, "Longitudinal acoustic modes and Brillouin-gain spectra for $\mathrm{GeO}_{2}$-doped-core single-mode fibers," $J$. Opt. Soc. Amer. B, Vol. 6, p. 1167, 1989.

[17] T. Kurashima, T. Horigushi, and M. Tateda, "Thermal effects on the Brillouin frequency shift in jacketed optical silica fibers," Appl. Opt., vol. 29 , p. $2219,1990$.

[18] A. S. Pine, "Brillouin scattering study of acoustic attenuation in fused quartz," Phys. Rev., vol. 185, p. 1187, 1969.

[19] T. Horiguchi, T. Kurashima, and M. Tateda, "Tensile strain dependence of Brillouin frequency shift in silica optical fibers," IEEE Photon. Technol. Lett., vol. 1, p. 107, 1989.
[20] M. Niklès, L. Thévenaz, and P. Robert, "Simple distributed fiber sensor based on Brillouin gain spectrum analysis," Opt. Lett., vol. 21, p. 758, 1995.

[21] _ "Simple distributed temperature sensor based on Brillouin gain spectrum analysis," in Proc. OFS'94 Conf., Glasgow Scotland, SPIE Publication, 1994, vol. 2360, pp. 138-141.

[22] "Measurement of the distributed-Brillouin-gain spectrum in optical fibers by using a single laser source," in Proc. Optical Fiber Commun. OFC'94 Conf., OSA Publication, San Jose, CA, paper WF1, 1994, p. 89.

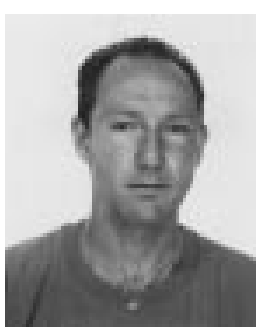

Marc Niklès received the Dipl.-Ing. degree in microtechnology from the Swiss Federal Institute of Technology of Lausanne (EPFL), Lausanne, Switzerland, in 1989. He received the Ph.D. degree from the Electrical Engineering Department at EPFL in 1997.

In 1990, he joined the Metrology Laboratory of the EPFL, where he developed metrological facilities for characterizing integrated optical devices and applications using optical signal processing. For his doctoral research, he investigated stimulated Brillouin scattering in optical fibers and the relevant applications to distributed fiber optic sensors.

Luc Thévenaz, for a photograph and biography, see p. 982 of the June 1997 issue of this JOURNAL.

Philippe A. Robert, for a photograph and biography, see p. 982 of the June 1997 issue of this JOURNAL. 